\title{
L'ADMINISTRATION FRANÇAISE ET L'UE-LE RÔLE DE LA REPRÉSENTATION PERMANENTE DE LA FRANCE AUPRÈS DES INSTITUTIONS EUROPÉENNES
}

\author{
$U D K: 327.82(44: 4-6 E U)$ \\ 321:061.1 EU \\ DOI: $10.31141 /$ zrpfs.2020.57.135.23 \\ Prethodno znanstveno priopćenje \\ Primljeno: 1. prosinca 2019.
}

\begin{abstract}
U radu se prikazuje uloga stalnog predstavništva Francuske (PR) u europskim institucijama. Ističe se kako je stalno predstavništvo povjereno karijernim diplomatima na visokoj razini koji pripadaju "mreži" stručnjaka specijaliziranih za europske poslove (stručnjaci za europska pitanja čine svojevrsnu aristokraciju među francuskim karijerskim diplomatima). Nadalje se navodi i analizira kako je uloga predstavništva vrlo složena zbog raznolikosti predmeta kojima se bave. Na kraju se zaključuje kako je francuski stalni predstavnik uvijek bio karijerni dilomat, za razliku od nekih država članica koje su ponekad imenovale bivše ministre.
\end{abstract}

Ključne riječi: stalna predstavništva Francuske u EU, europske institucije

Les ambassades multilatérales sont particulières puisque, à la différence des ambassades bilatérales, en général pour un Etat beaucoup plus nombreuses- à l'exception de micro-Etats qui parfois se bornent à une ambassade auprès de l'ONU-, elles ne sont pas seulement accréditées auprès d'une entité extérieure- en l'occurrence une organisation internationale ou régionale- mais encore participent aux décisions de cette entité. En effet, le représentant permanent et ses collaborateurs représentent leur gouvernement au sein des institutions ou des organes de cette organisation internationale ( le conseil de sécurité de l'ONU, le conseil atlantique ou le conseil de l'UE...). En général, les postes diplomatiques multilatéraux sont assez primés dans la carrière notamment les postes auprès des organisations internationales les plus importantes, c'est-à-dire, pour la France, l'ONU- compte-tenu du siège permanent au sein du Conseil de sécurité-, l'UE- ce qui est le cas pour tous les Etats membres de l'UE compte-tenu du caractère très particulier de l'UE, organisation régionale sui generis ayant atteint un degré d'intégration inédit, voire l'OTAN, même si cette dernière organisation s'interroge sur son avenir depuis la chute du mur de Berlin puis l'avènement de Donald Trump...

En ce qui concerne la représentation permanente(RP) de la France auprès de l'UE, elle est confiée à un diplomate de haut niveau, expert des questions européennes lesquelles ont toujours été particulières notamment par leur degré de complexité et de diversité dans les matières traitées. Les experts en questions européennes forment 
une sorte d'aristocratie parmi les diplomates français de carrière ; ils appartiennent à un « réseau » d'experts spécialistes des affaires européennes. Le représentant permanent a toujours été un diplomate de carrière, à la différence de certains Etats membres qui y ont parfois nommés des anciens ministres. En revanche, dans le cas français comme dans le cas de la plupart des RP, l'ambassadeur diplomate de carrière et le représentant permanent adjoint sont accompagnés de conseillers issus non seulement de la carrière diplomatique mais aussi de la plupart des autres ministères, leur nombre et leur diversité d'origine s'accroissant au fur et à mesure du développement des domaines d'intervention de la CEE, puis de la CE et enfin de l'UE.

Quelques mots d'histoire : les traités CEE et CEEA signés en mars 1957 disposaient (articles 151 et 121) que le règlement intérieur des Conseils pouvait prévoir la constitution d'un comité formé de représentants des Etats membres dont les Conseils détermineraient la mission et les compétences. Une semaine après l'entrée en vigueur des 2 traités, les 7 et 8 janvier 1958, les six ministres des affaires étrangères reconnurent l'opportunité de nommer aussitôt que possible des représentants permanents- évidemment nommés par chaque Etat membre- auprès des Communautés. Le 25 janvier 1958, le Conseil décidait également la création d'un COPEPER -comité des représentants permanents - qui serait intégré de facto au sein des institutions des Communautés européennes, au sein du Conseil. L'ambivalence de la RP éclatée entre le national, en tant que représentant d'un Etat membre, et le communautaire, en tant que participant à l'élaboration et la négociation de textes européens au sein du COPEPER, apparaissait posée dès l'origine.

Une croissance régulière des effectifs : la Représentation permanente française est une structure interministérielle, comprenant aujourd'hui une centaine de conseillers sur un total de 200 agents- soit 5 fois plus qu'en 1965- dont un tiers est issu du corps diplomatique( ces derniers suivent les relations extérieures de l'UE, les élargissements quand il y des négociations d'élargissement, les relations avec le Parlement et la presse, la PESC ainsi que certains dossiers plus techniques) personnellement j'avais été chargé un temps de la politique régionale- aujourd'hui appelée politique de cohésion- et de la politique de la pêche avant de devenir « Antici », c'est-à-dire chargé notamment de la préparation de l'ordre du jour du Coreper ( $2^{\text {ème }}$ partie) et de rendre compte des travaux du Conseil européen ; un diplomate ayant rang d'ambassadeur représente la France au sein du comité pour la politique de sécurité commune ( COPS aujourd'hui présidé par un fonctionnaire désigné par le haut représentant pour les affaires étrangères et la politique de sécurité, Mme Mogherini et bientôt M. Borrell ) . Les deux autres tiers des conseillers de la RP sont issus d'un nombre croissant de ministères, dont l'économie et les finances, l'agriculture, l'intérieur, les affaires sociales, la défense... Souvent un membre du Conseil d'Etat y est détaché en tant que conseiller juridique de la RP. L'AN et le Sénat disposent aussi d'un représentant auprès de l'UE hébergés au sein de la RP.

Interface entre la capitale et les institutions de l'Union européenne, la Représentation permanente joue un rôle central dans les communications entre 
Paris et Bruxelles, la conduite des négociations au sein des instances du Conseil de l'Union européenne et les relations avec les autres institutions, en particulier le Parlement européen et la Commission européenne.

Sa mission est d'une part de promouvoir et défendre les positions nationales auprès des institutions européennes( 1) et d'autre part d'informer au mieux la capitale sur l'état des questions européennes (2):

\section{1 - défendre et promouvoir les positions françaises :}

- la Représentation permanente participe à Bruxelles à l'élaboration et à la conduite de l'ensemble des politiques de l'Union européenne : marché intérieur, agriculture, pêche, consommation, tourisme, transports, télécommunications, emploi, industrie, santé, recherche, environnement, éducation, relations extérieures, questions budgétaires et financières, justice et affaires intérieures....

Dans ce cadre, les conseillers de la Représentation permanente expriment les positions de la France dans les instances ( multiples groupes de travail du Conseil ) préparant les réunions du Conseil des Ministres. La Représentation permanente assiste les membres du gouvernement lors des réunions du Conseil des ministres de l'Union européenne ainsi que le Président de la République et son équipe lors des Conseils européens.

Les travaux du Conseil sont préparés par le Comité des Représentants permanents (Coreper) dans ses deux formations : d'une part, le Coreper 2, celui des ambassadeurs représentants permanents, qui prépare les travaux du Conseil dans ses formations affaires étrangères, affaires économiques et financières, affaires générales et justice et affaires intérieures et, d'autre part, le Coreper 1 où les Etats sont représentés par leur représentant permanent adjoint - pour la France c'est aussi un diplomate, contrairement à d'autres Etats membres qui confient ce poste à un fonctionnaire issu d'un « ministère technique », c'est le cas de l'Allemagne et du Royaume-Uni, qui prépare les autres formations du Conseil : agriculture et pêche, compétitivité, éducation, jeunesse, culture et sport, emploi, politique sociale et consommateurs, environnement et transports, télécommunications et énergie. Chacun de ces deux se réunissent chaque semaine. Le Coreper surveille et coordonne également les travaux des comités- comité de coordination pour l'espace de liberté, de sécurité et de justice, comité économique et financier et des sous-gouverneurs de banque centrale composé des directeurs du trésor et des sous-gouverneurs de banque centrale traditionnellement très autonome-, COPS précité- et groupes de travail composés de fonctionnaires des Etats membres, diplomates des RP ou fonctionnaires venus des capitales, qui préparent au niveau technique les dossiers qui sont soumis au Coreper puis au Conseil, voire au Conseil européen pour les questions les plus sensibles sur lesquelles les ministres n'auraient pu se mettre d'accord.

La Représentation permanente est en relation avec tous les acteurs impliqués dans les processus de décision ; elle présente et explique les positions françaises aux membres de la Commission ( cabinets des commissaires, DG) et du Parlement européen. 
Elle travaille étroitement, depuis l'entrée en vigueur du traité de Lisbonne en 2009, avec le Service européen pour l'action extérieure que dirige le haut Représentant ( Mme Federica Mogherini puis M.Josep Borrell avec la nouvelle Commission) et appuie la lente montée en puissance de ce service qui est supposée conjuguer et intégrer la culture de la Commission, du Secrétariat général du Conseil et des Etats membres pour tenter de faire une synthèse des différentes politiques européennes dans leur dimension externe qui complétera l'action des diplomaties nationales. On sait combien cette genèse est complexe dans la mesure où les intérêts et priorités de politique extérieure des 27/28 diffèrent souvent, y compris parfois entre la France et l'Allemagne ou entre l'Europe du nord et l'Europe méditerranéenne.

\section{2 - informer la capitale sur l'état des discussions à Bruxelles :}

En sens inverse, la Représentation permanente informe quotidiennement de façon précise la capitale sur l'état des discussions européennes sur les questions en cours et, le cas échéant, sollicite les instructions nécessaires. Pour la France, ces instructions parviennent du Secrétariat général des Affaires européennes $(S G A E)$, placé auprès du Premier ministre, dont Y. Gounin et Mme Lahore viennent de vous entretenir. La RP peut participer par vidéoconférence aux réunions interministérielles présidées par le SGAE. La RP assiste le SGAE dans son rôle de garant de l'unité et de la cohérence de la position française au sein de l'Union européenne dans l'ensemble des enceintes européennes, les ministères tendant à défendre des intérêts parfois catégoriels qu'il faut parfois arbitrer dans un cadre plus général ( exemple du ministère de l'agriculture s'agissant de telle réforme de la PAC susceptible d'affecter les intérêts de telle catégorie d'agriculteurs ou du ministère du budget s'agissant du budget européen qu'il convient absolument de ne pas accroitre). C'est ici que la RP, à même de mieux connaître l'état des positions des institutions ( Commission, Parlement) et des principaux Etats membres concernés, peut contribuer à la formalisation de positions réalistes et équilibrées tenant compte des priorités essentielles.

Le RP participe aux réunions de préparation du Conseil affaires étrangères présidée à Paris par le ministre avant chaque Conseil.

\section{3 - La Représentation permanente est également en contact avec tous les autres intervenants intéressés par les questions européennes :}

- parlementaires nationaux, collectivités locales ( la plupart des régions disposent de bureaux à Bruxelles) ;

- représentants des entreprises ( pôle « entreprises et coopération »). Dans le respect des règles de concurrence, ce pôle informe les entreprises françaises sur les dossiers européens et les opportunités offertes par les programmes communautaires. Il propose des informations en ligne permettant d'accéder à des fiches sur l'aide extérieure de l'UE et de préparer les appels d'offres et les appels à propositions de la Commission. Le Pôle apporte des conseils individuels sur demande (messagerie, téléphone, entretien à la RP), participe à des actions collectives (séminaires d'information en France et à Bruxelles, 
rencontres avec des délégations d'entreprises, ateliers), oriente les opérateurs vers les interlocuteurs de la Commission, fournit de l'information opérationnelle aux organismes relais vers les entreprises. Le Pôle suit le Comité Consultatif Accès au marché (MAAC) et les groupes de travail sectoriels (MAWG) aux côtés des fédérations professionnelles et des grandes entreprises concernées. Il exerce une veille active sur l'évolution des obstacles à l'accès aux marchés tiers (barrières non tarifaires), en liaison avec le réseau diplomatique français ainsi que sur la mise en œuvre des accords commerciaux. L'action du Pôle porte sur la défense des intérêts des entreprises françaises dans des domaines variés : marchés publics sur financements européens (fonds structurels) ou même sur financements nationaux (question des aides d'Etat) ; propriété intellectuelle; licences et homologations ; concurrence ; etc... Le Pôle agit en étroite concertation avec les conseillers spécialisés de la Représentation permanente et avec les interlocuteurs concernés à la Commission.

- organisations syndicales, associations et ONG, chercheurs, think tanks et le public au sens large (étudiants notamment).

La RP participe au débat public en étant en contact étroit avec les journalistes, en s'ouvrant au public en général sur place et par son site internet (http://www.rpfrance. eu) et en suivant les principaux débats d'idées sur les questions européennes.

Elle veille à la place des Français et de la langue française dans les institutions européennes ( 38\% des documents de la Commission en 1996 étaient rédigés en français et seulement 5\% en 2011, respectivement pour l'anglais $45 \%$ et $80 \%$ ) .

\section{La RP en période de présidence semestrielle :}

La dernière présidence française remonte au second semestre 2008 mais c'était avant l'entrée en vigueur du traité de Lisbonne si bien que le Président de la République présidait alors le Conseil européen et le ministre des affaires étrangères le conseil des affaires étrangères ce qui n'est plus le cas depuis 2009 avec la création de la fonction de président permanent du CE, M.Tusk puis Charles Michel à compter d'octobre 2019 et le renforcement du rôle du haut représentant pour la politique étrangère. En période de présidence, la RP doit veiller à faire avancer les dossiers retenus prioritaires par la capitale tout en recherchant activement les compromis avec les autres Etats pour les faire aboutir. Selon le secrétariat du Conseil( site consilium.europa), « pour y parvenir, la présidence doit jouer le rôle d'un intermédiaire intègre et neutre ».

Concrètement, l'exercice de la présidence implique des renforts en conseillers et des communications accrues avec les ministères parisiens et la coordination interministérielle (SGAE) ainsi que la présidence de la République (Conseil européen) et avec les autres représentations permanentes, la Commission et le Parlement (trilogue) et le président du Conseil européen. Il faut sans cesse s'informer sur les priorités de chacun des autres Etats membres, et du PE et de la Commission afin de les rallier aux compromis nécessaires pour faire avancer les priorités de la présidence. 
Les traités ne reconnaissent que peu de responsabilité à la présidence semestrielle : elle convoque le Conseil ( art 237 TFUE) par son initiative ou celle d'un autre Etat membre ou de la Commission, elle établit l'ordre du jour provisoire du Conseil, fait procéder au vote et signe les règlements, directives et décisions du Conseil au même titre que le président du PE lorsqu'il s'agit d'actes législatifs adoptés selon la procédure législative ordinaire( codécision).Mais dans la pratique, la présidence peut jouer un rôle plus significatif. Si dans le passé chaque présidence devait en début de semestre établir son programme et le présenter devant le Parlement européen, désormais un programme des activités du Conseil pour 18 mois est proposé par les 3 pays amenés à présider successivement le Conseil- « le trio »-, en consultation avec le haut représentant, le président du Conseil européen et le président de la Commission européenne. Des arrangements sont possibles entre les ministres de ces 3 pays qui permettraient par exemple à l'un des 3 pays de présider les travaux relatifs à tel dossier pendant une période plus longue que six mois. Dans le dialogue avec la Commission et le Parlement, la présidence joue souvent un rôle d'intermédiaire et cherche des compromis permettant au «triangle interinstitutionnel» de fonctionner sans à-coups. L'exercice de certaines présidences ayant peu d'appétence européenne ( Tchéquie en 2009, Hongrie en 2011) a pu être problématique. Si lors des révisions successives des traités, le principe de la présidence tournante chaque semestre a parfois été discuté, les Etats restent attachés aux présidences semestrielles, même si les inconvénients sont connus : brièveté des six mois ce qui a notamment justifié la création de la fonction pérenne de président du Conseil européen. La présidence semestrielle traduit le principe d'égalité des Etats quelle que soit la taille de leur population et le poids de leur vote au conseil.

Ainsi la Finlande préside le conseil de l'UE de juillet à décembre 2019, la Croatie de janvier à juin 2020 puis l'Allemagne de juillet à décembre 2020. Ce «trio » a mis au point un programme détaillé ( $\mathrm{cf} 30$ novembre 2018 14518/18) qui couvre une période complexe puisqu'une nouvelle législature vient de commencer ses travaux et une nouvelle commission se met en place à l'automne et qu'un nouveau cadre financier pluriannuel pour la période 2021-2027 doit être adopté à l'unanimité. Les suites du feuilleton du Brexit ou de la crise migratoire ne manqueront pas de compliquer davantage encore l'exercice présidentiel.

La France a présidé depuis 1995 trois fois l'Union européenne (en 1995, 2000 et juillet-décembre 2008). La prochaine présidence française est prévue pour le premier semestre 2022.Je me souviens que, si la présidence française avait préparé longtemps à l'avance son programme, en réalité le déclenchement de la crise des sub-prime en 2008 puis la guerre russo-géorgienne en août avaient en partie bouleversé le plan prévu. L'exercice d'une présidence implique donc une complète préparation incluant des objectifs stratégiques mais aussi un plan de travail imposé par le calendrier ainsi qu'une capacité d'adaptation aux circonstances imprévues qui ne manqueront pas de surgir. 


\section{FRENCH ADMINISTRATION AND THE EUROPEAN ROLE OF FRANCE'S PERMANENT REPRESENTATION REGARDING EUROPEAN INSTITUTIONS}

The role of France's constant representation in European institutions is shown in this paper. That constant representation is entrusted to career diplomats is at a high level is pointed out who belong to "a network" of experts specialised for European jobs (experts for European issues make up their own kind of aristocracy among French career diplomats). Furthermore, the role of representation being very complex due to the variedness of topics being dealt with is noted and analysed. Finally, the conclusion is that France's permanent representative has always been a career diplomat, in contrast to some member states which have sometimes nominated former ministers.

Key words: France's permanent representation in the EU, EU institutions 